\title{
Dental deans should give dentists fair access to Royal College exams
}

Surgeons have stepped up their call for dental deans to give dentists access to Royal College exams.

The British Association of Oral and Maxillofacial Surgeons (BAOMS) is arguing that this would help advance dentists' careers and support those who wanted to join the oral surgery specialis list.

Currently, there are hundreds of dentists with oral surgery skills who work in hospitals and in dental practices across the UK.

However, unlike doctors in similar medical posts who can apply to their regulator the General Medical Council (GMC) for a Certificate of Eligibility for Specialist Registration (CESR) based on knowledge and skills, the General Dental Council (GDC) only allows applications to the oral surgery specialist list on the basis of academic qualifications and research.

As part of a CESR portfolio in surgery, doctors who are not in training programmes can apply for the FRCS (Fellowship of the Royal College of
Surgeons) exam in their specialty if they can supply three supporting references.

Dentists not in training programmes have no similar route. Exam regulations for the Membership in Oral Surgery and Intercollegiate Specialty Fellowship Exam rule them ineligible and discriminate in favour of specialty trainees and those on Masters programmes.

Vinita Shekar and Parminder Shoker, both hospital dentists who represent this group of dentists on the BAOMS Council and in the Royal College of Surgeons of England (RCSEng), said: 'We are frustrated that we do not have access to the Membership in Oral Surgery (MOral Surg) or the Intercollegiate Specialty Fellowship Exam (ISFE).

'Doctors in our position can sit the FRCS exam, but dentists who want to sit oral surgery exams to advance their careers or help them apply to get on the specialist list are excluded.'

The issue was also the focus of a paper ${ }^{1}$ published on 2 April 2019 in the Royal College of Surgeons of England's Faculty Dental Journal.
The authors highlighted their concerns over the steady reduction in the number of specialists in the GDC Oral Surgery specialist list. There was a decline from 794 specialists on the list in 2007 to 724 in 2017 - a 9\% fall over 11 years.

BAOMS Chair Patrick Magennis, one of the paper's authors, said: 'It's not about passing an exam as a shortcut onto the specialist list, but having a way to demonstrate knowledge.

'I am disappointed that the dental deans do not change the regulations to give these key workers in OMFS departments and skilled dentists working on the high-street this important opportunity.

'FRCS exams for surgeons are robust enough to accept all comers. Other dental specialty exams accept candidates from outside training. Why should oral surgery exams be so different?'

\section{References \\ 1. Fullarton M, Jadun S, Begley A, Magennis P. The oral surgery specialist: what will happen as the 'grandfa- thers' disappear? Fac Dent J. 2019; 10: 52-57.}

\section{BDA launches updated tool for special care and paediatric dentistry}

The BDA has issued an updated version of its case mix tool ${ }^{1}$, promising to build on what it describes as an unrivalled approach to scoring patient complexities and which contains new criteria covering paediatric dentistry.

The tool was first launched in 2008 to help dentists score the complexity of patients requiring special care dentistry and has become the gold standard for such scoring, according to six identifiable criteria. Results are used in commissioning and contract matters as well as in wider epidemiological work.

The six independent criteria that, either solely or in combination, indicate a measurable level of patient complexity include:

- Ability to communicate

- Ability to co-operate

- Medical status

- Oral risk factors

- Access to oral care

- Legal and ethical barriers to care.

Its decision to significantly update the tool, said the trade union, followed calls from commissioners to use the tool to measure paediatric dental patient complexities, hence the new criteria geared around the needs of young patients, following input from the British Society of Disability and Oral Health and British Society of Paediatric Dentistry.

Its definitions already included for adult patients have also been revised.

Discussions with IT suppliers are underway and the BDA will be producing training on the new tool later in 2019.

Although CDS dentists are its prime users, the tool can be used by general dental practitioners or secondary care providers who are treating patients requiring special care and paediatric dentistry.

Chair of the BDA's England Community Dental Services Committee, Charlotte Waite, said: 'Since its inception, BDA case mix has demonstrated to commissioners the challenges and value of the work community and special care dentists do. This new version will take this approach to the next level.
'For over a decade, BDA case mix has been used by individual dentists to demonstrate the complexity of their caseload and clinical directors to benchmark the clinics within their areas. This new version builds on a tried and tested system which has supported colleagues operating in all four countries of the UK.

'The NHS Long Term Plan pledges links to learning disability and autism services, and a holistic approach to care. It's an approach we take on a daily basis, empowered by BDA case mix, and now children can benefit fully with a system designed around their unique needs.

'The sheer complexity and skill of the services we deliver can often be lost on health officials and commissioners, and that's where BDA case mix comes into its own.'

\section{References}

1. BDA. Case Mix 2019. Available at https://www.bda. org/casemix (accessed April 2019). 